\title{
Clinical and epidemiological profile of cerebral venous thrombosis
}

\section{A Multicenter retrospective study in Aseer Region Saudi Arabia}

Mohammed S. Alqahtani, MBBS, Adel A. Alhazzani, MD,FRCPC,FAAN, Ibraheem Alnaemy, MD, Saeed A. Alqahtani, MD, Tariq M. Alahmari, MBBS, Abdulaziz M. Alqarni, MBBS, Ibrahim A. Alburaidi, MBBS, Mohammed A. Alqahtani, MBBS, Saleh M. Alqahtani, MBBS, Moayad A. Zarbh, MBBS, Yasser wassel, MD, Jaber Alfaif, MD, Ahmed Elhadad, MD.

\begin{abstract}

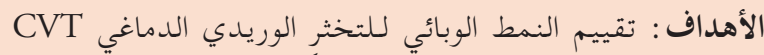

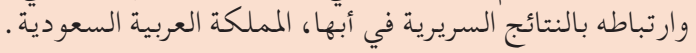

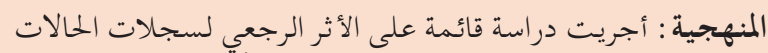

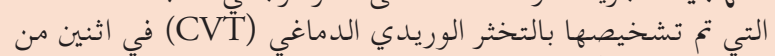

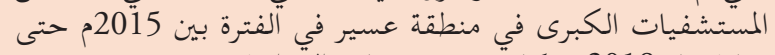

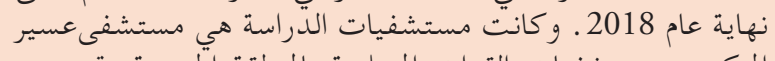

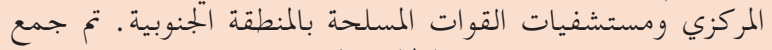

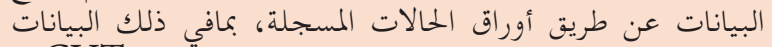

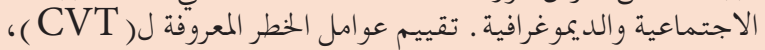

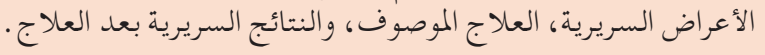
النتائج : شملت الدراسة 119 مريضا يعانون من ( CVT (CVT) )، والذين

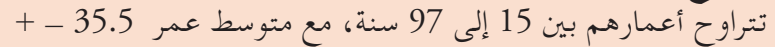

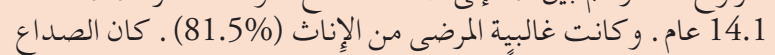

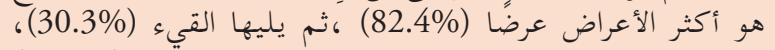

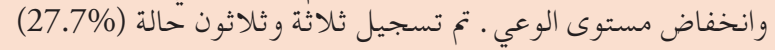

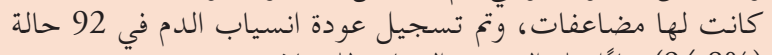
(94.8\%) بناءًا على التصوير الوعائي للدماغ توداغ.

الحلاصة : كشفت الدراسة أن أغلب حالات التخثر الوريدي

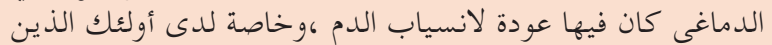

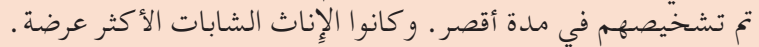

Objectives: To assess the epidemiological pattern and correlates with the clinical outcome of Cerebral venous thrombosis (CVT) in Abha, Kingdom of Saudi Arabia.

Methods: A retrospective record-based cohort design was conducted including all patients admitted with diagnosis of CVT in 2 main tertiary hospitals in Aseer Region between 2015 to the end of 2018. The study hospitalswere Aseer Central Hospital and Armed Forces
Hospitals Southern Region. The data were collected by structured data sheets, including sociodemographic data. Assessment of known risk factors for CVT, clinical presentation, treatment received, and clinical outcome after treatment were extracted.

Results: The study included 119 patients with CVT, whose ages ranged from 15 to 97 years, with a mean age of 35.5-+14.1 years. Majority of the patients were females $(81.5 \%)$. Headache was the most presenting $(82.4 \%)$ symptom, followed by vomiting $(30.3 \%)$ and a decreased level of consciousness. Thirty-three cases $(27.7 \%)$ had complications, and recanalization was recorded among 92 cases $(94.8 \%)$ based on follow up vascular imaging.

Conclusion: The study revealed that most of the cases of CVT had favorable clinical outcome and recanalization, especially those who had a shorter duration untildiagnosis. Young females were the most affected group.

Neurosciences 2020; Vol. 25 (5):380-385 doi: $10.17712 / n s j .2020 .5 .20200028$

From the Neurology Unit (Alqahtani MS, alfaifi, wassel), Department of Internal Medicine, Department of Radiology (Alahmari, Elhaddad), Armed Forces Hospital Southern Region, from the Department of Neurology (Alqahtani, Alnaemy), Aseer Central Hospital, from the Department of Neurology (Alqarni), King Khalid University, and from the Department of Medical (Alburaidi, Alqahtani MA, Alqahtani $S M$, zarbah), King Khalid University, Abha, and from the Neurology Unit (Alhazzani), Department of Medicine, College of Medicine, King Saud university, Riyadh, Kingdom of Saudi Arabia

Received 28th February 2020. Accepted 2nd July 2020.

Address correspondence and reprint request to: $D r$. Adel Alhazzani, Neurology Unit, Department of Medicine, College of Medicine, King Saud university, Riyadh, Kingdom of Saudi Arabia. E-mail: aalhazzani2@ksu.edu.sa

ORCID ID: https://orcid.org/0000-0002-9884-2243 
$\mathrm{C}$ erebral venous thrombosis (CVT) accounts for $0.5 \%$ to $1 \%$ of all strokes. Younger women are at high risk among other groups. ${ }^{1}$ The most presenting symptoms are headaches, focal neurological deficit, a decreased level of consciousness (LOC), seizures, or even intracranial hypertension with no focal neurological signs. ${ }^{2,3}$ Daif et al. reported a frequency in Saudi Arabia of 7 cases per 100,000 hospital patients. ${ }^{4}$ A prospective population-based survey conducted in the Isfahan region of Iran, between 2001 and 2004, reported an annual incidence of CVT of 12.1 per million people. ${ }^{5}$

A study of the anatomy of the venous system is essential to help evaluate patients with CVT, The venous circulation of the brain is drained to the superficial and the deep venous system. The drained blood runs into the major dural sinuses: the superior sagittal sinus (SSS), the inferior sagittal sinus (ISS), the lateral sinus (LS), the cavernous sinus, the straight sinus, and finally the internal jugular vein (IJV). Difficulties in diagnosis in some cases is due to high proportion of anastomoses between these 2 cerebral venous systems. ${ }^{6,7}$

Multiple predisposing factors of CVT exist. Many cases have been linked to inherited and acquired thrombophilia, pregnancy, puerperium, infection, and malignancy. Infarctions due to CVT are often hemorrhagic and associated with vasogenic edema. ${ }^{8}$ MRI with T1, T2, fluid-attenuated inversion recovery, and $\mathrm{T}^{*}$ sequences, in addition to magnetic resonance angiography, are the best diagnostic tools. D-dimer concentrations are elevated in the majority of patients, but normal D-dimers do not exclude CVT, especially in patients who present with only headaches. ${ }^{9}$ Treatment should start as soon as the diagnosis is confirmed. Treatment methods include reversing the underlying cause, control of seizures and intracranial hypertension, and antithrombotic therapy. Anticoagulation is the pillar of acute and sub-acute treatment for CVT. ${ }^{8,9}$

This research aims to identify the patterns and different risk factors associated with CVT, in addition to assessing the clinical outcome for CVT cases with treatment modalities. To the best of our knowledge, no similar study has been done in aseer region uptodate.

Methods. A retrospective record-based cohort design was conducted targeting all the accessible medical files of patients who had CVT in 2 main tertiary hospitals

Disclosure. Authors have no conflict of interests, and the work was not supported or funded by any drug company. in Aseer Region between 2015 to the end of 2018 with Confirmed Diagnosis of CVT by either CT Venogram or MR Venogram in adults 14 years and older, the study excluded pediatric population and clinical suspected CVT without vascular imaging. The study hospitals were Aseer Central Hospital and Armed Forces Hospitals Southern Region, Southern Saudi Arabia. They are the main tertiary hospitals in Aseer Region, to which nearly all cases are referred to receive treatment. The data were collected by structured data sheets, including socio demographic data (age, gender, work, marital status, and nationality). Assessment of known risk factors for CVT, clinical presentation, treatment received, and clinical outcome after treatment were extracted. The data were extracted from the hospital records after obtaining ethical approval.

Data analysis. Data was collected, reviewed and validated, then analyzed using IBM SPSS version 22 (SPSS, Inc. Chicago, IL) statistical software. All statistical analyses were carried out using a two-tailed test. A $p$-value less than 0.05 was considered to be statistically significant. Descriptive analysis based on frequency and percentage distributions was carried

Table 1 - Personal data of patients with Cerebral Venous Thrombosis, Aseer Region-Southern of Saudi Arabia.

\begin{tabular}{lrl}
\hline Personal data & $\mathbf{n}$ & $(\%)$ \\
\hline Gender & 22 & $(18.5)$ \\
Male & 97 & $(81.5)$ \\
Female & & \\
Age in years & & $(35.3)$ \\
$<30$ years & 42 & $(35.3)$ \\
$30-39$ & 35 & $(29.4)$ \\
40+ & & \\
Marital status & 34 & $(28.6)$ \\
Single & 85 & $(71.4)$ \\
Married & & \\
Nationality & 107 & $(89.9)$ \\
Saudi & 12 & $(10.1)$ \\
Non-Saudi & & \\
Educational level & & $(15.1)$ \\
Illiterate & 18 & $(22.7)$ \\
Basic & 44 & $(37.0)$ \\
Secondary & 30 & $(25.2)$ \\
Bachelor & & \\
Work & 43 & $(36.1)$ \\
Working & 63 & $(52.9)$ \\
Not working & 13 & $(10.9)$ \\
Student & & \\
Area & 78 & $(65.5)$ \\
High altitude & 41 & $(34.5)$ \\
Sea level & &
\end{tabular}




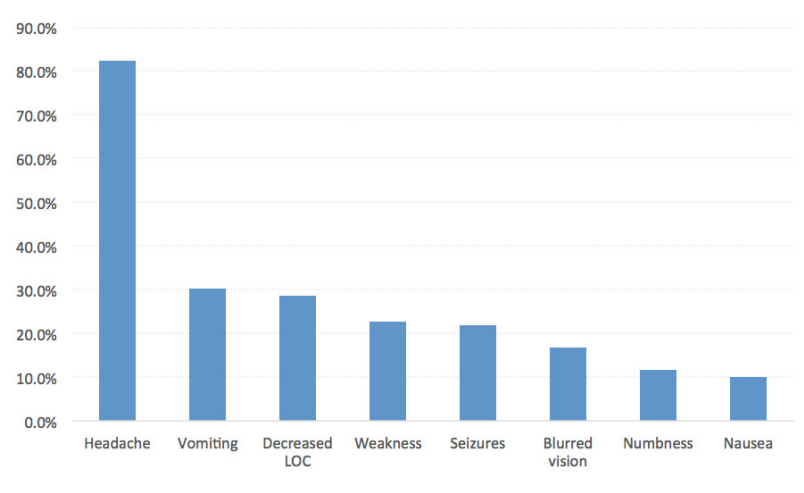

Figure 1 - Clinical presentation of patients with Cerebral Venous Thrombosis, Aseer Region-Southern of Saudi Arabia.

out for all variables, including demographic data, risk factors, and patterns and outcomes of CVT. Univariant relations between patients' biodemographic and clinical data with CVT clinical outcomes were determined based on exact probability tests because of small frequencies.

Results. The study included 119 patients with CVT whose ages ranged from 15 to 97 years, with a mean age of $35 \pm 14$ years. Majority of cases 97 (81.5\%) were females, of whom $71.4 \%$ were married. Education of a secondary degree or higher was recorded for $62.2 \%$ of the cases, $52.9 \%$ of the cases were not working, and $10.9 \%$ of the cases were students. Exactly $65.5 \%$ of the participants resided in high-altitude areas (Table 1).

As for the clinical presentation of CVT recorded among the study cases (Figure 1), headache was the most frequent $(82.4 \%)$ symptom, followed by vomiting $(30.3 \%)$, decreased LOC (28.6\%), weakness $(22.7 \%)$, and nausea $(10.1 \%)$.

Table 2 demonstrates the pattern of CVT among the study cases. Forty-two percent of the patients presented within 2 days from symptoms onset. The CTV was carried out for $52.9 \%$ of the cases, and CT with MRI was needed for $58 \%$ of the cases. About 39\% of the cases had 3-5 hours until undergoing CT, and intra cerebral hemorrhage was recorded for $41.9 \%$ of the cases. Also, $28.4 \%$ of the cases underwent MRI after 24 hours, while the majority of the cases $(84.9 \%)$ were diagnosed within two days from hospitalization. Imaging recorded that superior sagittal sinus were the most commonly involved in isolation $(21.8 \%)$ followed by transverse sinus, both sinuses were affected in 13.4 $\%$ of the cases. The CVT was on the left side for $58 \%$ of the cases.

Family history was significant for thromboembolic diseases in more than on forth $28.6 \%$ of the cases, and $11.8 \%$ of them were diabetic. Recurrent CVT was seen
Table 2 - Pattern of CVT among affected patients, Aseer Region-Southern of Saudi Arabia.

\begin{tabular}{|c|c|c|}
\hline Pattern of CVT & No & $(\%)$ \\
\hline \multicolumn{3}{|c|}{ The duration of the symptoms before presentation(days) } \\
\hline $1-2$ & 50 & $(42)$ \\
\hline $3-6$ & 38 & $(31.9)$ \\
\hline $7+$ & 31 & $(26.1)$ \\
\hline \multicolumn{3}{|l|}{ Diagnostic imaging } \\
\hline CTV & 63 & $(52.9)$ \\
\hline MRV & 56 & $(47.1)$ \\
\hline \multicolumn{3}{|l|}{ Imaging done } \\
\hline $\mathrm{CT}$ & 24 & $(20.2)$ \\
\hline MRI & 26 & $(21.8)$ \\
\hline Both & 69 & $(58.0)$ \\
\hline \multicolumn{3}{|l|}{ Time to do CT (hours) } \\
\hline $1-2$ & 21 & $(22.6)$ \\
\hline $3-5$ & 36 & $(38.7)$ \\
\hline $6-10$ & 16 & $(17.2)$ \\
\hline$>10$ & 20 & $(21.5)$ \\
\hline \multicolumn{3}{|l|}{ Initial image findings } \\
\hline Normal & 27 & $(29.0)$ \\
\hline Hemorrhage ICH & 39 & $(41.9)$ \\
\hline Edema or infarct & 27 & $(29.0)$ \\
\hline \multicolumn{3}{|l|}{ Time to do MRI } \\
\hline$<6$ hours & 21 & $(22.1)$ \\
\hline $6-12$ & 24 & $(25.3)$ \\
\hline $13-24$ & 23 & $(24.2)$ \\
\hline After 24 hours & 27 & $(28.4)$ \\
\hline \multicolumn{3}{|l|}{ Average time of diagnosis (days) } \\
\hline $1-2$ & 101 & $(84.9)$ \\
\hline $3-7$ & 18 & $(15.1)$ \\
\hline \multicolumn{3}{|l|}{ Location } \\
\hline Superior Sagittal Sinus & 26 & $(21.8)$ \\
\hline Transverse Sinus & 21 & $(17.6)$ \\
\hline Sigmoid sinus & 12 & $(10.1)$ \\
\hline Superior Sagittal + transverse & 16 & $(13.4)$ \\
\hline Transverse + sigmoid & 32 & $(26.9)$ \\
\hline Multiple sinuses & 12 & $(10.1)$ \\
\hline Right & 50 & $(42.0)$ \\
\hline Left & 69 & $(58.0)$ \\
\hline $\begin{array}{r}\text { CVT - Cerebral venous t } \\
\text { resonance venography, ICH } \\
\text { computerized }\end{array}$ & $\begin{array}{l}\text { s, MR } \\
\text { bral h } \\
\text { hy sc }\end{array}$ & $\begin{array}{l}\text { legnetic } \\
\text { hage, CT - }\end{array}$ \\
\hline
\end{tabular}

for $5 \%$ of the cases, while history of DVT founded in $8.4 \%$. About one third $31 \%$ of the female cases took OCP, and $44.3 \%$ were found to have hypercoaguable state (Table 3).

As for the clinical assessment of cases on admission, Table 4 illustrates that $95.8 \%$ of the cases achieved the good and favorable clinical outcome (0-2) based on Modified Rankin Scale (mRs), 2 cases were critical and 
Table 3 - Risk factors of CVT among affected patients, Aseer RegionSouthern of Saudi Arabia.

\begin{tabular}{|c|c|c|}
\hline Risk factors & No & $(\%)$ \\
\hline \multicolumn{3}{|c|}{ Family history of DVT/ stroke } \\
\hline No & 85 & $(71.4)$ \\
\hline Yes & 34 & $(28.6)$ \\
\hline \multicolumn{3}{|c|}{ History of hypercoagulopathy state } \\
\hline Yes & 43 & $(44.3)$ \\
\hline No & 54 & $(55.7)$ \\
\hline \multicolumn{3}{|l|}{ Co-morbidity } \\
\hline No & 80 & $(67.2)$ \\
\hline $\mathrm{DM}$ & 14 & (11.8) \\
\hline HTN & 9 & $(7.6)$ \\
\hline Others & 16 & $(13.4)$ \\
\hline \multicolumn{3}{|l|}{ Past history of } \\
\hline CVT & 6 & $(5.0)$ \\
\hline PE & 8 & $(6.7)$ \\
\hline DVT & 10 & $(8.4)$ \\
\hline \multicolumn{3}{|c|}{ Other risk factors } \\
\hline OCP & 37 & $(31.1)$ \\
\hline Pregnancy & 2 & $(1.7)$ \\
\hline Surgery & 8 & $(6.7)$ \\
\hline Post-partum & 13 & $(10.9)$ \\
\hline
\end{tabular}

one of them died after he went for surgical intervention. Only 23 patients $(19.3 \%)$ were stayed for more than 2 weeks in the hospital, and $71.4 \%$ had IV heparin on admission. Fifteen cases (12.6\%) were discharged on NOAC.

Regarding the clinical outcome, 33 cases $(27.7 \%)$ had complications, and recanalization was recorded for 92 cases $(94.8 \%)$ based on follow-up imaging.

Finally, relating the clinical outcome with the case factors (Table 5) revealed that all cases who had symptoms for 1-2 days only were completely recovered, compared with $92.3 \%$ of those who had symptoms for 7 days or more (with a statistical significance of $p$-value=.048). Also, $86.2 \%$ of the patients who had complications recovered, compared with $98.5 \%$ of those who did not $(p=.012)$. All other factors were not statistically significantly related to the clinical outcome.

Discussion. Cerebral venous sinus thrombosis (CVST) occurs as result of limitation of venous flow due to presence of a blood clot in the dural venous sinuses, which drain blood from the brain. Symptoms may include headache, abnormal vision, any of the symptoms of stroke such as weakness of the face and limbs on one side of the body, and seizures. ${ }^{10-12}$ The diagnosis is usually made by computed tomography (CT scan) or magnetic resonance imaging (MRI) to
Table 4 - Admission data of patients with Cerebral Venous Thrombosis, Aseer Region-Southern of Saudi Arabia.

\begin{tabular}{lll}
\hline Admission data & $\mathrm{n}$ & $(\%)$ \\
\hline Modified Rankin Scale & $(\mathrm{mRs})$ & \\
$\mathrm{mRs} 5-6$ & 2 & $(1.7)$ \\
$\mathrm{mRs} 3-4$ & 3 & $(2.5)$ \\
$\mathrm{mRs} 0-2$ & 114 & $(95.8)$ \\
Admission days & & \\
$1-6$ & 46 & $(38.7)$ \\
$7-14$ & 50 & $(42.0)$ \\
$15+$ & 23 & $(19.3)$ \\
Initial management & & \\
IV heparin & 85 & $(71.4)$ \\
SC heparin & 34 & $(28.6)$ \\
Discharge medication & & \\
Warfarin & 102 & $(85.7)$ \\
NOAC & 15 & $(12.6)$ \\
Still on medication & & \\
Yes & 87 & $(73.1)$ \\
No & 32 & $(26.9)$ \\
\hline
\end{tabular}

demonstrate obstruction of the venous sinuses. ${ }^{10,12,13}$ Treatment is typically with anticoagulants (medication that suppresses blood clotting) such as low molecular weight heparin. ${ }^{14}$ Thrombolytic (enzymatic destruction of the blood clot) is rarely used. The disease may be complicated by raised intracranial pressure, which may warrant surgical intervention such as the placement of a shunt. ${ }^{15}$ rarely endovascular interventions may be used in selected cases that does not respond to medical therapy. ${ }^{16}$

The current study aimed to assess the epidemiological pattern of CVT among cases, including site, affected sinuses, laterality, and risk factors. Also, we aimed to detect the clinical outcome for the cases in relation to different factors, including treatment received. The study revealed that most of the CVT cases were young females below the age of 40 years, with the most recorded risk factors are history of miscarriage and taking oral contraceptive pills (OCP). Furthermore, high altitude was also recorded more among CVT cases, maybe due to the nature of the Abha area or the association of high altitude with high blood viscosity. Headache, vomiting, and deteriorated consciousness were the most recorded symptoms, which are all nonspecific and may be confused with any less serious clinical cases, resulting in delayed seeking for medical advice; this was actually the situation as more than half of the cases needed 3 days or more before consulting medical advice. As for imaging, more than half of the cases required both CT 
Table 5 - Predictors of recanalization among patientswith Cerebral Venous Thrombosis, Aseer Region-Southern of Saudi Arabia.

\begin{tabular}{|c|c|c|c|c|c|}
\hline \multirow[t]{3}{*}{ Predictor } & \multicolumn{4}{|c|}{ Finding on CT follow up } & \multirow{3}{*}{$P$-value } \\
\hline & \multicolumn{2}{|c|}{ Still thrombus } & \multicolumn{2}{|c|}{ Recanalization } & \\
\hline & $\mathbf{n}$ & $(\%)$ & $\mathrm{n}$ & $(\%)$ & \\
\hline \multicolumn{6}{|l|}{ Gender } \\
\hline Male & 1 & $(5.9)$ & 16 & $(94.1)$ & \multirow{2}{*}{.881} \\
\hline Female & 4 & $(5.0)$ & 76 & $(95.0)$ & \\
\hline \multicolumn{6}{|l|}{ Age in years } \\
\hline$<30$ years & 0 & $(0.0)$ & 32 & $(100)$ & \multirow{3}{*}{.272} \\
\hline $30-39$ & 3 & $(7.9)$ & 35 & $(92.1)$ & \\
\hline $40+$ & 2 & $(7.4)$ & 25 & $(92.6)$ & \\
\hline \multicolumn{6}{|l|}{ Area } \\
\hline High altitude & 3 & $(4.9)$ & 58 & $(95.1)$ & \multirow{2}{*}{.891} \\
\hline Sea level & 2 & $(5.6)$ & 34 & $(94.4)$ & \\
\hline \multicolumn{6}{|c|}{ The duration of the symptoms before presentation(days) } \\
\hline $1-2$ & 0 & $(0.0)$ & 44 & $(100)$ & \multirow{3}{*}{$.048^{*}$} \\
\hline $3-6$ & 3 & $(11.1)$ & 24 & $(88.9)$ & \\
\hline $7+$ & 2 & $(7.7)$ & 24 & $(92.3)$ & \\
\hline \multicolumn{6}{|l|}{ Location } \\
\hline Superior Sagittal Sinus & 2 & $(9.5)$ & 19 & $(90.5)$ & \multirow{6}{*}{.139} \\
\hline Transverse Sinus & 0 & $(0.0)$ & 17 & $(100)$ & \\
\hline Sigmoid sinus & 2 & $(18.2)$ & 9 & $(81.8)$ & \\
\hline Superior Sagittal + Transverse & 0 & $(0.0)$ & 14 & $(100)$ & \\
\hline Transverse + Sigmoid & 0 & $(0.0)$ & 25 & $(100)$ & \\
\hline Superior Sagittal + Transverse + Sigmoid & 1 & $(11.1)$ & 8 & $(88.9)$ & \\
\hline \multicolumn{6}{|l|}{ Site } \\
\hline Right & 2 & $(4.9)$ & 39 & $(95.1)$ & \multirow{2}{*}{.916} \\
\hline Left & 3 & $(5.4)$ & 53 & $(94.6)$ & \\
\hline \multicolumn{6}{|l|}{ Initial management } \\
\hline IV heparin & 5 & $(7.2)$ & 64 & $(92.8)$ & \multirow{2}{*}{.144} \\
\hline SC heparin & 0 & $(0.0)$ & 28 & $(100)$ & \\
\hline \multicolumn{6}{|l|}{ Discharge medication } \\
\hline Warfarin & 5 & $(5.3)$ & 89 & $(94.7)$ & \multirow{2}{*}{.682} \\
\hline NOAC & 0 & $(0.0)$ & 3 & $(100.0)$ & \\
\hline \multicolumn{6}{|l|}{ Average time of diagnosis (days) } \\
\hline $1-2$ & 5 & $(6.0)$ & 78 & $(94.0)$ & \multirow{2}{*}{.346} \\
\hline $3-7$ & 0 & $(0.0)$ & 14 & $(100)$ & \\
\hline \multicolumn{6}{|l|}{ Still on medication } \\
\hline Yes & 4 & $(5.6)$ & 68 & $(94.4)$ & \multirow{2}{*}{.762} \\
\hline No & 1 & $(4.0)$ & 24 & $(96.0)$ & \\
\hline Complications & & & & & \\
\hline Yes & 4 & $(13.8)$ & 25 & $(86.2)$ & $.012^{*}$ \\
\hline No & 1 & $(1.5)$ & 67 & $(98.5)$ & $.012^{\top}$ \\
\hline P - Exact probability te & & 05 (signifi & cant) & & \\
\hline
\end{tabular}

and MRI to be diagnosed, and hemorrhage was the most recorded finding, while in one third of the cases the imaging was normal. The superior sagittal sinus were the most commonly involved in isolation followed by transverse sinus, especially on the left side.

As for risk factors, family history was dominant, followed by DM in general, but a history of having hypercoagulopathy state and OCP were the most dominant factors for females. Recanalization was recorded for majority of the cases as only 5 cases showed failed recanalization on follow-up imaging. Outcomes of patients who were discharge on NOAC 
were comparable to those on warfarin but the number was small (15 cases $12.6 \%)$ to draw solid conclusion. A for comparison with the study was conducted by Algahtani et $\mathrm{al}^{17}$ in Saudi Arabia to analyze the clinical patterns, etiologies, treatment, and outcome of CVST in 2 major cities of Saudi Arabia: Jeddah and Al-Baha. The researchers reported 92 adults and 19 children with CVST. Among the adults, females were the most affected, while also more males than females were affected. The mean age of onset was 29.5 years. The most recorded clinical presentations were headache, focal neurologic deficits, seizures, papilledema, and a decreased level of consciousness. The main risk factors recorded were pregnancy/puerperium, antiphospholipid antibody syndrome, oral contraceptive pills, malignancy, and infections. The superior sagittal sinus $(24.3 \%)$ was the most common site of CVST. Seventy-four patients recovered completely, 23 patients recovered partially, and 10 patients died. Bad prognostic factors included incurable co-morbid conditions, late presentation, and status epilepticus. All are consistent with the current study findings. Results of our study are in line with previously published data from western part of Saudi Arabia in terms of clinical presentations and risk factors, however outcome in our study population was slightly better. This could be due to increased awareness and earlier diagnosis and treatment, as nearly half of the patient establish diagnosis within 48 hrs.

The limitations of this study as a retrospective study include selection bias and potential for non-measurable confounders; therefore, multicenter prospective studies are needed in the future. Overall, CVT is not a rare disease and may lead to death or disability if not diagnosed and treated promptly. Diagnosis can be challenging due to variable and nonspecific symptoms therefore high index of suspension is required and utilization of readily available diagnostic imaging is mandatory to confirm the diagnosis. A better understanding of the epidemiological pattern, including the most frequent presentations and associated risk factors, may help in reducing this management gap.

In conclusions, the study revealed that most of the cases of CVT had favorable clinical and radiological outcomes, in particular when diagnosed within 48 hours. Young females living at high altitude were the most affected group. Using OCP and presence of hypercoagulopathy state were the most prominent risk factors among females. More than half of the cases needed more than 48 hours for presentation and diagnosis which indicates the need to raise awareness of target population about this condition.
Acknowledgment. We would like to thank ProofreadingServices.com, for English language editing. We acknowledge that first and second Authors contributed equally to this manuscript.

\section{References}

1. Danwang C, Mazou TN, Tochie JN, Tankeu R, Bigna JJ. Global epidemiology and patterns of cerebral venous thrombosis: a systematic review and meta-analysis protocol. BMJ Open 2018; 8: e019939.

2. Tadi P, Behgam B, Baruffi S. Cerebral Venous Thrombosis. Treasure Island (FL); Statpearls Publishing: 2020.

3. Ashjazadeh N, Haghighi AB, Poursadeghfard M, Azin $\mathrm{H}$. Cerebral venous-sinus thrombosis: a case series analysis. Iran J Med Sci 2011; 36: 178-182.

4. Daif A, Awada A, Al-Rajeh S, Abduljabbar M, Al Tahan AR, Obeid T, et al. Cerebral venous thrombosis in adults: a study of 40 cases from Saudi Arabia. Stroke 1995; 26: 1193-1195.

5. Zia A, Wasay M, Kaul S. Epidemiology of cerebral venous thrombosis in asian countries. Pakistan Journal of Neurological Sciences 2014; 3: Article 13.

6. Kiliç T, Akakin A. Anatomy of Cerebral Veins and Sinuses. Front Neurol Neurosci 2008; 23: 4-15.

7. Alvis-Miranda HR, Castellar-Leones SM, Alcala-Cerra G, Moscote-Salazar LR. Cerebral sinus venous thrombosis. $J$ Neurosci Rural Pract 2013; 4: 427-438.

8. Silvis SM, Middeldorp S, Zuurbier SM, Cannegieter SC, Coutinho JM. Risk Factors for Cerebral Venous Thrombosis. Semin Thromb Hemost 2016; 42: 622-631.

9. Bousser MG, Ferro JM. Cerebral venous thrombosis: an update. Lancet Neurol 2007; 6: 162-170.

10. Al Rawahi B, Almegren M, Carrier M. The efficacy and safety of anticoagulation in cerebral vein thrombosis: A systematic review and meta-analysis. Thrombosis Research 2018; 169: 135-139.

11. Stam J. Thrombosis of the cerebral veins and sinuses. $N$ Engl J Med 2005; 352: 1791-1798.

12. Cumurciuc R, Crassard I, Sarov M, Valade D, Bousser MG. Headache as the only neurological sign of cerebral venous thrombosis: a series of 17 cases. J Neurol Neurosurg Psychiatry 2005; 76: 1084-1087.

13. Einhäupl K, Stam J, Bousser MG, De Bruijn SFTM, Ferro JM, Martinelli I, et al. EFNS guideline on the treatment of cerebral venous and sinus thrombosis in adult patients. Eur J Neurol 2010; 17: 1229-1235.

14. Ferro JM, Canhão P, Marie-Germaine B, Stam J. Cerebral vein and dural sinus thrombosis in elderly patients. Stroke 2005; 36 : 1927-1932.

15. Smith R, Hourihan MD. Investigating suspected cerebral venous thrombosis. BMJ 2007; 334: 794-795.

16. Ilyas A, Chen CJ, Raper DM, Ding D, Buell T, Mastorakos P, et al. Endovascular mechanical thrombectomy for cerebral venous sinus thrombosis: a systematic review. J Neurointerv Surg 2017; 9: 1086-1092.

17. Algahtani HA, Abdu AP, Shami AM, Hassan AE, Madkour MA, Al-Ghamdi SM, et al. Cerebral venous sinus thrombosis in Saudi Arabia. Neurosciences (Riyadh) 2011; 16: 329-334. 